\title{
Level-set and Image Statistics for Pose Estimation of Satellites
}

\author{
Jian-Feng Shi ${ }^{1}$, Steve Ulrich ${ }^{2}$, Stéphane Ruel ${ }^{3}$ \\ ${ }^{1,2}$ Mechanical and Aerospace Engineering, Carleton University \\ 3135 Mackenzie, 1125 Colonel By Dr., Ottawa, Canada \\ jianfeng.shi@carleton.ca; steve.ulrich@ carleton.ca \\ ${ }^{3}$ Neptec Design Group Ltd. \\ 302 Legget Drive Suite 202, Kanata, Canada \\ sruel@neptec.com
}

\begin{abstract}
This paper investigates a level-set and image pixel probability-based method to compute pose estimation of satellites. The level-set segmentation technique elevates the two-dimensional image contours to a third dimension allowing easy handling of topology changes. The investigated method allows efficient feedback in the pose estimation process by combining level-set segmentation with pixel likelihood prior generated by a 3D model projection. This investigation provides an original matrix formulation of the energy partial derivative with respect to the pose parameters accounting for the pixel skew factor. Enhancement of the posterior map is provided using Otsu's thresholding and a flood-fill sequence. Results show the improvement reduces the degradation of the pixel probability and level-set contour generation error. This method was tested using colour and monochromatic monocular spacecraft images.
\end{abstract}

Keywords: Level-set Segmentation, Pose Estimation.

\section{Introduction}

Spacecraft relative pose estimation is an essential input to the larger guidance-navigation and controls pipeline. This investigation focuses on the navigation state estimation by computing pose from monocular camera images. Camera pose estimation is a core problem in computer vision. Selected methods using feature-based structures such as points, lines, ellipses and chains to free-form objects such as active contours and implicit surfaces are summarised by Lepetit, Fua [1] and Rosenhahn et al. [2,3]. Keypoints based on image corners [4,5] and extrema image blobs [6,7] can be either overly numerous which strains the computation resource or scarce under extreme lighting, viewpoint, and plainly surface-textured visual targets. Feature descriptor matching can be time consuming and erroneous under high affine transformations or extreme illuminations. Edge-based pose estimation [8] represent stable features to track with many invariant properties, but extracting relevant edge features and discarding the clutter lines requires a robust decision tool as the edge and feature point image can be cluttered with unstable and none-useful entities such as those shown in Fig. 1. On the other hand, region-based pose estimation uses invariant shape features with higher recognition properties. This investigation shall focus on the use of region-based methods for spacecraft pose estimation.

Region-based pose estimation approach evolved from image segmentation. In the seminal papers by Kass et al. [9], Mumford and Shah [10], the concepts of active contours, the minimisation of region smoothness and boundary length were introduced respectively. Caselles et al. [11], Chan and Vese [12] adapted implicit region boundary representation by using the level-set function [13]. The level-set method provides advantages of implicit boundary without specific parameterisation that can elegantly handle boundary topology changes such as splitting and merging. Additional contributions [14-16] infused the statistical likelihood of estimating the energy functional. Cremers [17] extended the ideas of using kernel PCA [18] and developed the use of affine flow fields [19] in the segmentation. Further developments in regional shape prior using pixelwise posteriors [20-22] was combined in solving 3D pose estimation. Consequently, the level-set segmentation and pose estimation complemented each other in a closed-loop framework where gradient descent can be applied [23-27].

Various advances have been made in the modernisation of the level-set based segmentation and pose estimation. Dambreville et al. [28] uses a 3D model as a projection to the 2D image shape prior and comparing the log-likelihood of Gaussian densities. Prisacariu and Reid used posterior membership probabilities for foreground and background pixel in support of real-time tracking [25,29]. Hexner and Hagege [26] proposed a framework for using local appearance model extending the PWP3D global region statistics. This local framework improved all aspects of scale, translation, and rotation. Tjaden et al. [30] proposed a pixel-wise optimisation strategy based on a Gaub-Newton-like algorithm using linearised twist for pose 


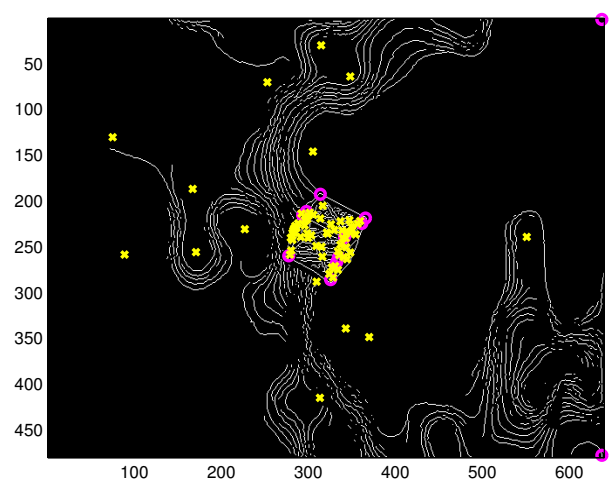

(a) CubeSat

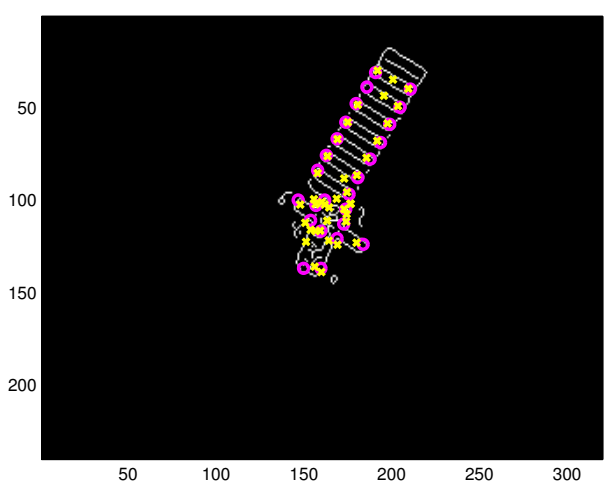

(b) EnviSat

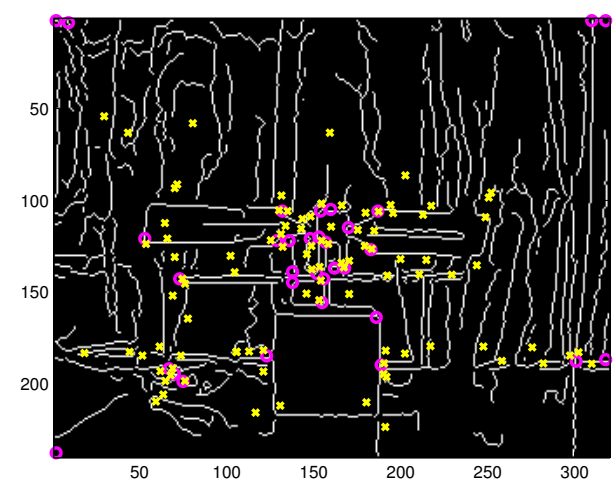

(c) RadarSat

Fig. 1: Canny edge lines in white, Harris corners in magenta circles, and SIFT keypoints in yellow crosses. When viewed with a non-illuminated space background in the case of the Envisat, the keypoints and edges are invariant inputs for pose estimation and tracking. Both line and keypoint features are cluttered and require classification of the feature descriptors when a rotating Earth background is in the image.

parameterisation. Tjaden et al. [27] also proposed a temporally consistent, local colour histogram as an object template descriptor allowing for a more precise level-set evolution.

This investigation is based on the works by Rosenhahn et al. [22], Brox et al. [23] Schmaltz [24], Prisacariu and Reid [25]. The contributions of this investigation are as follows:

1. The development of an original matrix formulation of the level-set energy partial derivative with respect to the pose parameters while accounting for the pixel-skew factor.

2. An enhancement to the probability posterior mask using image processing techniques to improve estimation stability.

This paper is organised as follows: Section 2 provides the methodology for the level-set based pose estimation technique and enhancements. Section 3 provides the images used in the test trials. Section 4 provides the results and discussions on the method and improvements. Finally, section 5 concludes this investigation.

\section{Framework and Methods}

Regional based pose estimation is achieved by the combined use of level-set segmentation and 3D model registration. Section 2.1 defines the basic notations. Section 2.2 provides an introduction of the level-set approach, the level-set energy formulation, and a method for pixel likelihood in computing the image segmentation. Section 2.3 provides a method for 3D model projection and object pose estimation.

\subsection{General Notation}

Given an input image $\mathbf{I}$ and the image domain $\Omega \subset \mathbb{R}^{2}$. The image pixel $\mathbf{x}$ with coordinates $(x, y)$ has a corresponding feature $\mathbf{y}$. This feature could be an integer pixel intensity, a colour vector, or some temporal state. The contour around the object of interest is denoted by $C$. The foreground region segmented by $C$ is denoted as $\Omega_{f}$, and the background is denoted as $\Omega_{b}$. The foreground and background regions has its own statistical appearance model, $P\left(\mathbf{y} \mid M_{i}\right)$ for $i \in\{f, b\}$, where $P$ is the probability density function computed by the image histogram. $\Phi$ is the level-set embedding function. Further description of $\Phi$ shall be provided in Sec. 2.2. The $H(z)$ and $\delta(z)$ are the smoothed Heaviside step function and the smoothed Dirac delta function respectively. A $3 \mathrm{D}$ point $\mathbf{X}_{c} \in \mathbb{R}^{3}$ with coordinates $\left(X_{c}, Y_{c}, Z_{c}\right)^{T}$ expressed in the camera frame $\overrightarrow{\mathscr{F}}_{c}$ can be obtained from the object point $\mathbf{X}_{b} \in \mathbb{R}^{3}$ expressed in the object's body frame $\overrightarrow{\mathscr{F}}_{b}$ with coordinates $\left(X_{b}, Y_{b}, Z_{b}\right)^{T}$. Using a rotation from $\overrightarrow{\mathscr{F}}_{b}$ to $\overrightarrow{\mathscr{F}}_{c}$ denoted by $\mathbf{R} \in S O(3)$ and a translation from $\overrightarrow{\mathscr{F}}_{c}$ to $\overrightarrow{\mathscr{F}}_{b}$ expressed in $\overrightarrow{\mathscr{F}}_{c}$ denoted by $\mathbf{t}=\left(t_{x}, t_{y}, t_{z}\right)^{T} \in \mathbb{R}^{3} . \mathbf{R}$ can be parameterised by the quaterion $\mathbf{q}=\left(q_{x}, q_{y}, q_{z}, q_{w}\right)^{T}=\left(\boldsymbol{\epsilon}^{T}, \eta\right)^{T}$, such that

$$
\mathbf{R}=\left(\eta^{2}-\epsilon^{T} \epsilon\right) \mathbf{1}+2 \epsilon \boldsymbol{\epsilon}^{T}-2 \eta \epsilon^{\times},
$$

where $\mathbf{1} \in \mathbb{R}^{3 \times 3}$ is the identity matrix. Note the sign direction of $q_{w}$ from Eq.(1) for the implementation under this investigation. The individual coordinates of $\left(\mathbf{t}^{T}, \mathbf{q}^{T}\right)^{T}$ is represented by $\gamma_{i}$ where $i \in\{1, \ldots, 7\}$. 


\subsection{Level-set Pose Estimation}

The level-set formulation [13] provides a simple mathematical framework for the implicit description of contour evolution. The merging, splitting, appearing, and disappearing of contours can be easily described by a higher dimensional entity $\Phi$ than by the explicit formulation of the curve entity $C$. For example, a contour in a two-dimensional image is defined by the zero level in a Lipschitz continuous function $\Phi$ in a three-dimensional surface. Formally, $C=\{(x, y) \in \Omega \mid \Phi(x, y)=0\}$. The level-set function $\Phi$ is evolved rather than directly evolving $C$. The subset of the level-set function is a signed distance function $d(\mathbf{x})$ defined as

$$
d(\mathbf{x})=\min _{\mathbf{x}_{i} \in C}\left|\mathbf{x}-\mathbf{x}_{i}\right|,
$$

hence, $|\nabla \Phi(x, y)|=1$. Figure 2 provides an example of the contour around the Envisat spacecraft shown in the normal image, the distance map, and a slope skewed level-set function for illustration. The level-set formulation of the piecewise constant

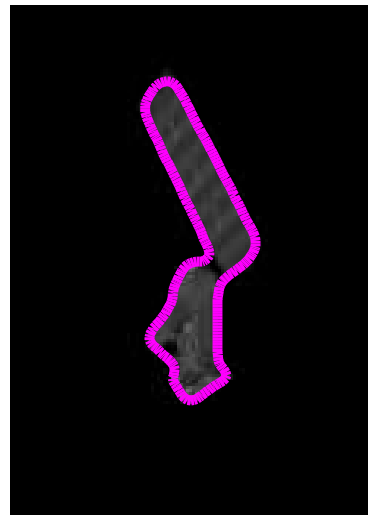

(a) Curvature Image in $2 \mathrm{D}$

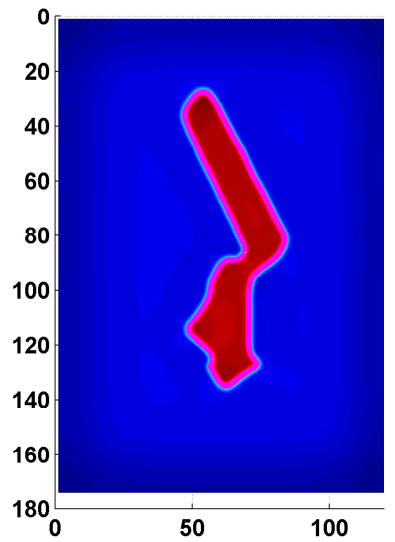

(b) Distance map representation

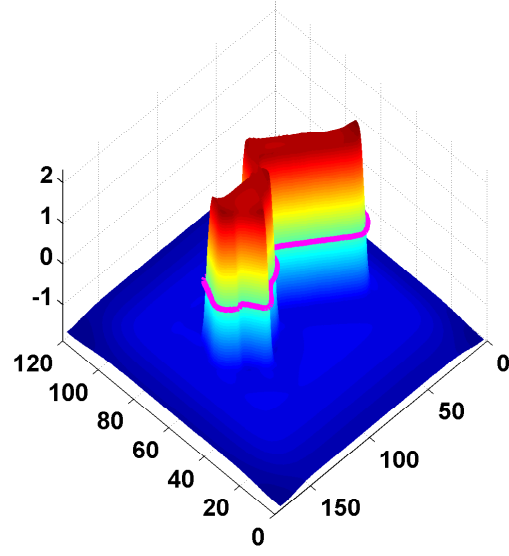

(c) Level-Set function in 3D

Fig. 2: The final state of a slope skewed level-set function. The level-set function evolves from some initial bounding shape and conforms to the Envisat spacecraft edge lines in the image plane. The absolute sign from Eq.(2) is omitted in the distance function for clear illustration.

Mumford-Shah functional $[10,12,31]$ that allows for the two-phase segmentation of an image $I: \Omega \rightarrow \mathbb{R}$ can be generated by minimising an energy function [32],

$$
E=\int_{\Omega_{f}} \alpha_{f}(\mathbf{I}(\mathbf{x}), C) d \Omega+\int_{\Omega_{b}} \alpha_{b}(\mathbf{I}(\mathbf{x}), C) d \Omega=\int_{\Omega}\left(H(\Phi) \alpha_{f}(\mathbf{x})+(1-H(\Phi)) \alpha_{b}(\mathbf{x})\right) d \Omega,
$$

where $\alpha_{i}$ for $i \in\{f, b\}$ is the likelihood of the pixel property, where $\alpha_{i}(\mathbf{x})=P\left(\mathbf{y} \mid M_{i}\right)$ and $i \in\{f, b\}$. Bibby and Reid [20] proposed an effective energy formulation as the posterior of each pixel's respective membership. Assuming pixel-wise independence, and replacing the integration with a summation of the contour log posterior probability, the energy becomes,

$$
E(\Phi)=-\log (P(\Phi \mid \mathbf{I}))=-\log \left(\prod_{\mathbf{x} \in \Omega}\left(H(\Phi) P_{f}+(1-H(\Phi)) P_{b}\right)\right)=-\sum_{\mathbf{x} \in \Omega} \log \left(H(\Phi) P_{f}+(1-H(\Phi)) P_{b}\right),
$$

and the foreground and background probabilities $P_{f}$ and $P_{b}$ are

$$
P_{i}=\frac{P\left(M_{i} \mid \mathbf{y}\right)}{P\left(M_{i}\right)}=\frac{P\left(\mathbf{y} \mid M_{i}\right)}{P\left(\mathbf{y} \mid M_{f}\right) P\left(M_{f}\right)+P\left(\mathbf{y} \mid M_{b}\right) P\left(M_{b}\right)},
$$

and $P\left(M_{i}\right)$ where $i \in\{f, b\}$, is the prior and can be computed by taking the normalised areas of the respective regions,

$$
P\left(M_{f}\right)=\sum_{\mathbf{x} \in \Omega} H(\Phi(\mathbf{x})), \quad P\left(M_{b}\right)=\sum_{\mathbf{x} \in \Omega}(1-H(\Phi(\mathbf{x}))) .
$$




\subsection{Pose Estimation}

The target object pose can be estimated directly using the energy functional as described in Eq.(4) by taking the partial derivative with respect to the individual pose parameters $\gamma_{i}$. This allows the evolution of the target boundary with respect to its pose rather than time. Let define $\partial(a) / \partial \gamma_{i}=a_{\gamma_{i}}, \nabla_{t}(a)=\left(a_{t_{x}}, a_{t_{y}}, a_{t_{z}}\right)^{T}$, and $\nabla_{q}(a)=\left(a_{q_{x}}, a_{q_{y}}, a_{q_{z}}, a_{q_{w}}\right)^{T}$. The energy partial derivative is

$$
E_{\gamma_{i}}=-\sum_{\mathbf{x} \in \Omega} \frac{P_{f}-P_{b}}{\left(P_{f}-P_{b}\right) H(\Phi)+P_{b}} \delta(\Phi)(\nabla \Phi)^{T} \mathbf{x}_{\gamma_{i}}
$$

where $\nabla$ is the image gradient over $\mathbf{x}$. The camera projection model can be used to relate the 3D model to the $2 \mathrm{D}$ image,

$$
\left[\begin{array}{l}
\mathbf{x} \\
1
\end{array}\right]=Z_{c}^{-1} \mathbf{K}\left[\begin{array}{ll}
\mathbf{1} & \mathbf{0}
\end{array}\right]\left[\begin{array}{cc}
\mathbf{R} & \mathbf{t} \\
\mathbf{0}^{T} & 1
\end{array}\right] \mathbf{X}_{b}=\left[\begin{array}{ccc}
f S_{x} & f S_{\theta} & o_{x} \\
0 & f S_{y} & o_{y} \\
0 & 0 & 1
\end{array}\right] \frac{\mathbf{X}_{c}}{Z_{c}}
$$

where $\mathbf{0} \in \mathbb{R}^{3}, \mathbf{K}$ is the intrinsic camera matrix, $\mathbf{X}_{c} / Z_{c}$ is the depth normalised object point observed and expressed from the camera frame, $f$ is the focal length of the camera, $S_{\theta}$ is the pixel skew scaling, $S_{i}$ and $o_{i}$ where $i \in\{x, y\}$ is the pixel scaling and image origin to center distance respectively. Equation (8) can be used to derive an expression for $\mathbf{x}_{\gamma_{i}}$ such that,

$$
\mathbf{x}_{\gamma_{i}}=\frac{f}{Z_{c}^{2}}\left[\begin{array}{c}
\mathbf{X}_{c}^{T}\left(S_{x} \mathbf{T}_{x}+S_{\theta} \mathbf{T}_{y}\right) \\
\mathbf{X}_{c}^{T}\left(S_{y} \mathbf{T}_{y}\right)
\end{array}\right]\left(\mathbf{X}_{c}\right)_{\gamma_{i}}
$$

where

$$
\mathbf{T}_{x}=\left[\begin{array}{ccc}
0 & 0 & -1 \\
0 & 0 & 0 \\
1 & 0 & 0
\end{array}\right], \quad \mathbf{T}_{y}=\left[\begin{array}{ccc}
0 & 0 & 0 \\
0 & 0 & -1 \\
0 & 1 & 0
\end{array}\right],
$$

The partial derivative of $\mathbf{X}_{c}$ with respect to the pose parameters $\gamma_{i}$ can be derived from the extrinsic translation and rotation of the body coordinates to the camera coordinates through $\mathbf{X}_{c}=\mathbf{R} \mathbf{X}_{b}+\mathbf{t}$, the partial derivative results are as follows,

$$
\nabla_{t} \mathbf{X}_{c}^{T}=\mathbf{1}, \quad \nabla_{q} \mathbf{X}_{c}^{T}=2\left[\begin{array}{lll}
\mathbf{A} \mathbf{X}_{b} & \mathbf{B} \mathbf{X}_{b} & \mathbf{C} \mathbf{X}_{b}
\end{array}\right]
$$

where

$$
\mathbf{A}=\left[\begin{array}{ccc}
0 & q_{y} & q_{z} \\
-2 q_{y} & q_{x} & -q_{w} \\
-2 q_{z} & q_{w} & q_{x} \\
0 & q_{z} & -q_{y}
\end{array}\right], \quad \mathbf{B}=\left[\begin{array}{ccc}
q_{y} & -2 q_{x} & q_{w} \\
q_{x} & 0 & q_{z} \\
-q_{w} & -2 q_{x} & q_{y} \\
-q_{z} & 0 & q_{x}
\end{array}\right], \quad \mathbf{C}=\left[\begin{array}{ccc}
q_{z} & -q_{w} & -2 q_{x} \\
q_{w} & q_{z} & -2 q_{y} \\
q_{x} & q_{y} & 0 \\
q_{y} & -q_{x} & 0
\end{array}\right]
$$

\section{Experimental Images and 3D Models}

The experimental images and internal 3D model used by the algorithm are a generic 1-U Cubesat, the Envisat, and the Radarsat model. These images and 3D models are illustrated in Fig. 3. Experimental images are generated by virtual cameras using the 3D Studio Max ${ }^{\circledR}$ software. The Envisat trials uses initial condition (IC) position $\left[\begin{array}{lll}x & y & z\end{array}\right]=\left[\begin{array}{lll}0 & 0 & 80\end{array}\right] \mathrm{m}$ and Euler angle pyr-sequence orientation [ roll pitch yaw $]=\left[\begin{array}{lll}0 & 0 & 0\end{array}\right]$ to $\left[\begin{array}{lll}0 & 0 & 360\end{array}\right]$ deg. The Radarsat model trials uses IC position [ $\left.\begin{array}{lll}0 & 0.05 & 0.7\end{array}\right] \mathrm{m}$ and Euler angle pyr-sequence orientation [ $\left[\begin{array}{llll}-90 & 180 & 0\end{array}\right]$ to [ $\left.-90 \quad 180 \quad 360\right]$ deg.

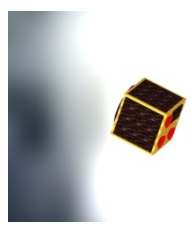

(a) Cubesat Image

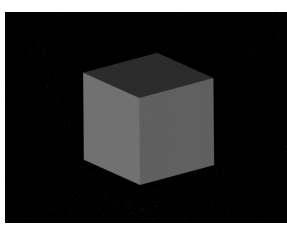

(b) Cubesat Model

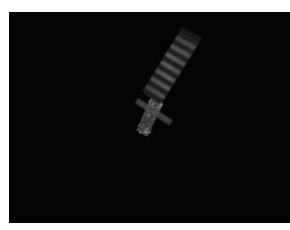

(c) Envisat Image

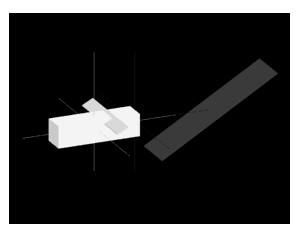

(d) Envisat Model

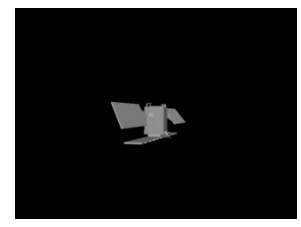

(e) Radarsat Image

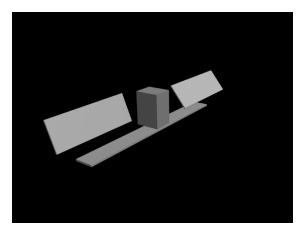

(f) Radarsat Model

Fig. 3: Experimental images and internal 3D models. 


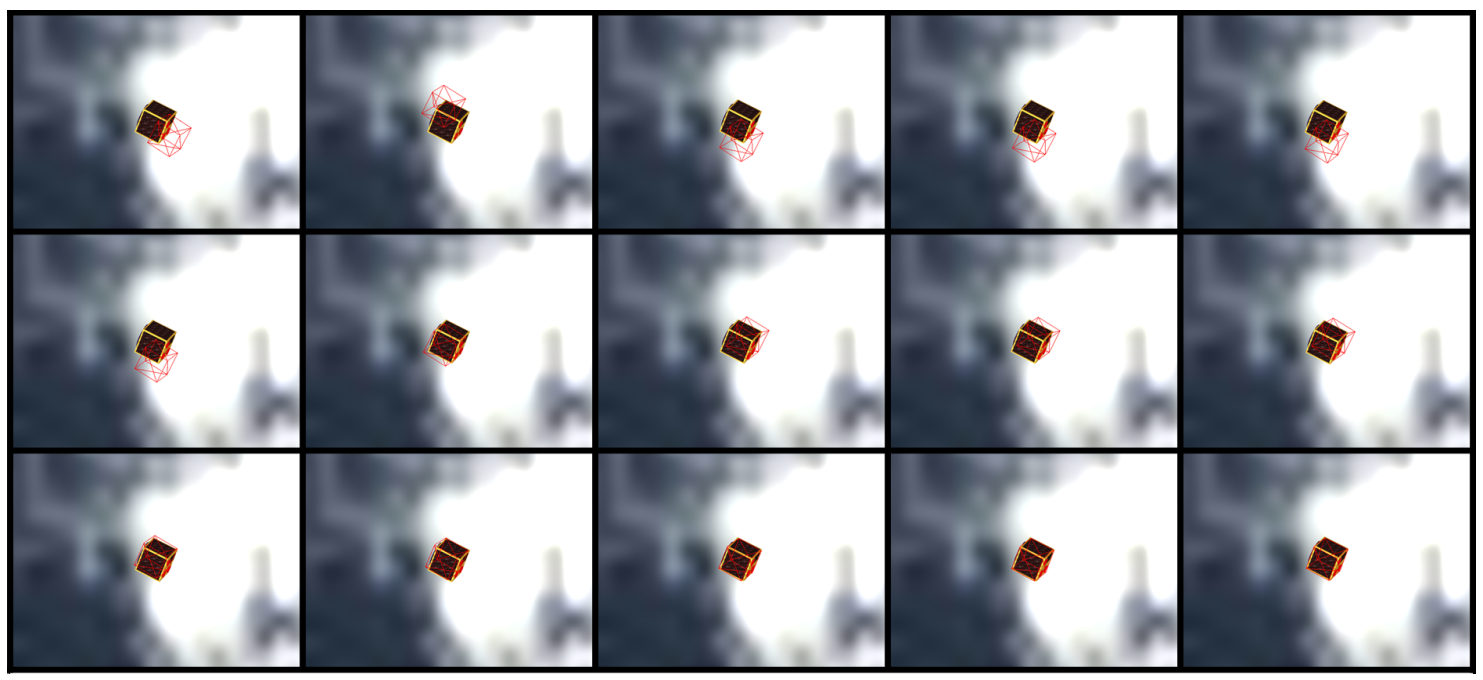

Fig. 4: Gradient descent results for a single image. For large initial offset between the model projection and the image, the gradient descent is performed by a coarse depth and lateral movement, followed by rotation (as shown by the first row), then by the same combination in fine step adjustments (as shown by the second row). Finally, even smaller step adjustments in all directions are applied simultaneously (as shown by the third row).

\section{Results and Discussions}

A sequence of gradient descent for the CubeSat pose estimation is shown in Fig. 4. Estimation typically starts with a large initial condition offset, and subsequent tracking is performed using the segmentation mask from the previous frame to define the foreground and background pixels. A process for step-size tunning was developed for efficient convergence from large initial misalignments. This iteration process is described in detail per Fig. 4. For each pose generation, the pixel foreground, and background probability posterior are computed and converted to a segmentation mask for the subsequent frame. For normal operations, statistical training is unnecessary for every frame; however, the likelihood needs to be refreshed periodically such that it is current to the observed scenery. The foreground target and the background are usually separable for colour images and images with dark space as the background. Grayscale images with relatively similar foreground and background contrast are much more difficult to classify using pixel distribution of the global image. For images with Earth backgrounds, contour error will creep in over time resulting in pose estimation failure. A remedy for reducing the error creep is to apply Otsu thresholding to the posterior map removing low probability regions and noise; this will result in a clean mask for the subsequent frame level-set estimation as shown in Fig. 5.

Degradation of the probability mask can occur if the sequential mask generated by the posterior is replaced with the firstframe a priori as input. This is because the training data histogram is corrupted by mixing foreground and background pixels from the wrong pose projection segmentation mask. This error exposes the primary weakness in using the level-set pose estimation method since it is strongly dependent on having current and separable pixel histograms between the foreground and the background. The Otsu thresholding of the posterior mask is further enhanced with an image fill process to reduce the estimation degradation. The improvement process is provided as follows: 1. Perform Otsu thresholding on the classified foreground posterior map in generating the segmentation mask. 2. Flood fill the segmentation mask with its background. 3. Invert the flood fill segmentation mask. 4. Combine the threshold segmentation mask with the inversed flood filled mask using the bitwise OR operation. The enhancement resulted in a cleaner segmentation mask as shown in Fig. 6.

Multi-sequence pose estimation for the CubeSat is shown in Fig. 7. The silhouette projection of the 3D model with enhancements was made to the posterior results to stabilises the level-set contour evolution. While the contour boundary tightly restricts the lateral and depth estimation, rotations of symmetrical bodies such as the CubeSat can have an error drift over the image sequence. This error is accumulated over many frames and can be difficult to jump out from the local minimum trap. Future developments shall focus on producing better orientation estimations by using perspective- $n$-point techniques internal to the foreground image. The results of the Envisat rotation sequence and pose estimation are provided in Fig. 8. Targets with non-symmetrical geometry has greater resilience to pose iteration and can allow for larger step-size. Results of the Radarsat model rotation sequence and pose estimation are provided in Fig. 9. The root sum squared (RSS) error of the position and orientation are provided in Fig. 10 for the envisat and radarsat trials from Fig. 8 and Fig. 9 respectively. Results show the highest error when segmented regions with minimum areas. Radarsat model exhibit higher relative error due to a combination of large out-of-plane view-point changes, smaller and closer model targets, and greater corruption to the region silhouette due to shadowing. While the core pose parameter for computation uses quaternions, floating point numerical precision near rotational singularities for orientation output displays requires careful attention. 


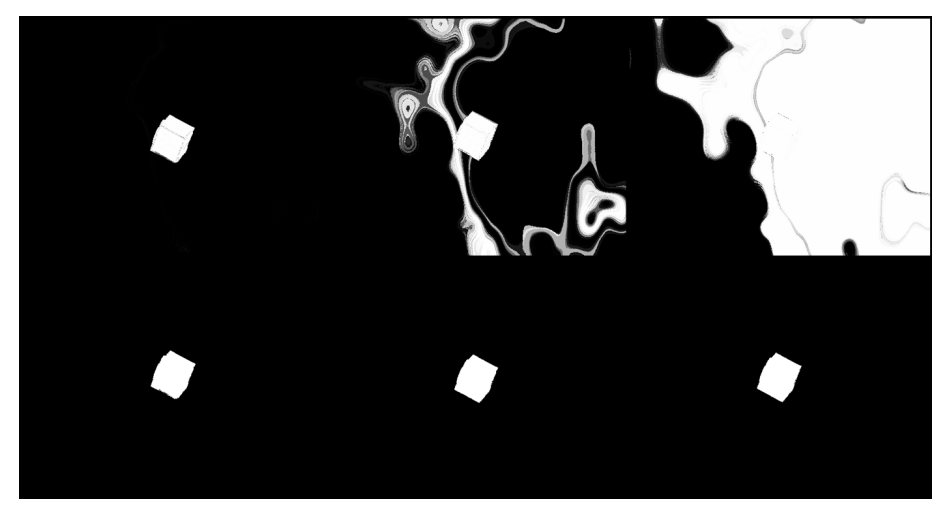

Fig. 5: Image sequence pose estimation uses the foreground pixel probability posterior generated from the previous frame. Leakage of background contour is amplified after three frames as shown by the first row. The segmentation mask is improved by using the proposed thresholding and fill technique as shown by the second row.

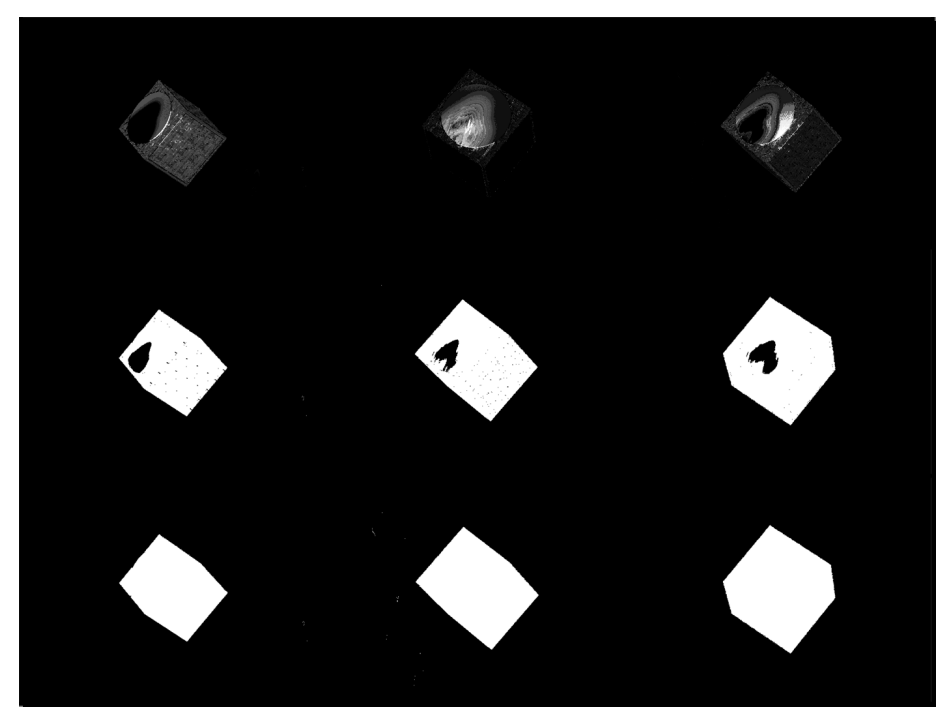

Fig. 6: The posterior of later frames is degraded over time if only using the a priori mask in generating the training histograms (shown by the first row). Using immediate previous frames and Otsu thresholding results in a stronger foreground posterior (shown by the second row). The additional filling of the segmentation mask improves the posterior results (shown by the third row).

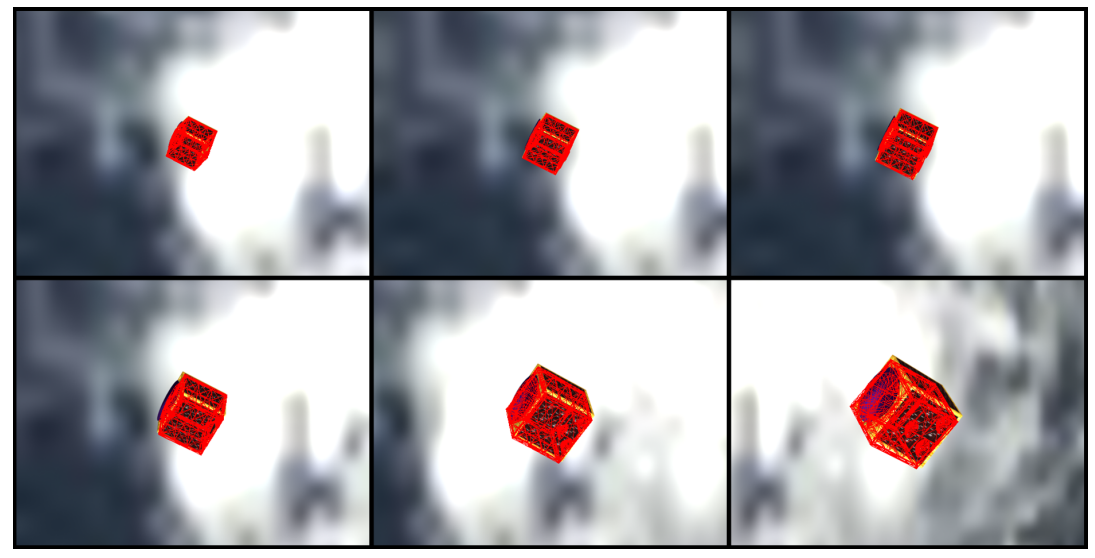

Fig. 7: Pose estimation results for multiple image frames from an approach maneuver. 3D projection matches the segmented target image with some error in the out-of-plane rotation that is difficult to infer by silhouette registration. 


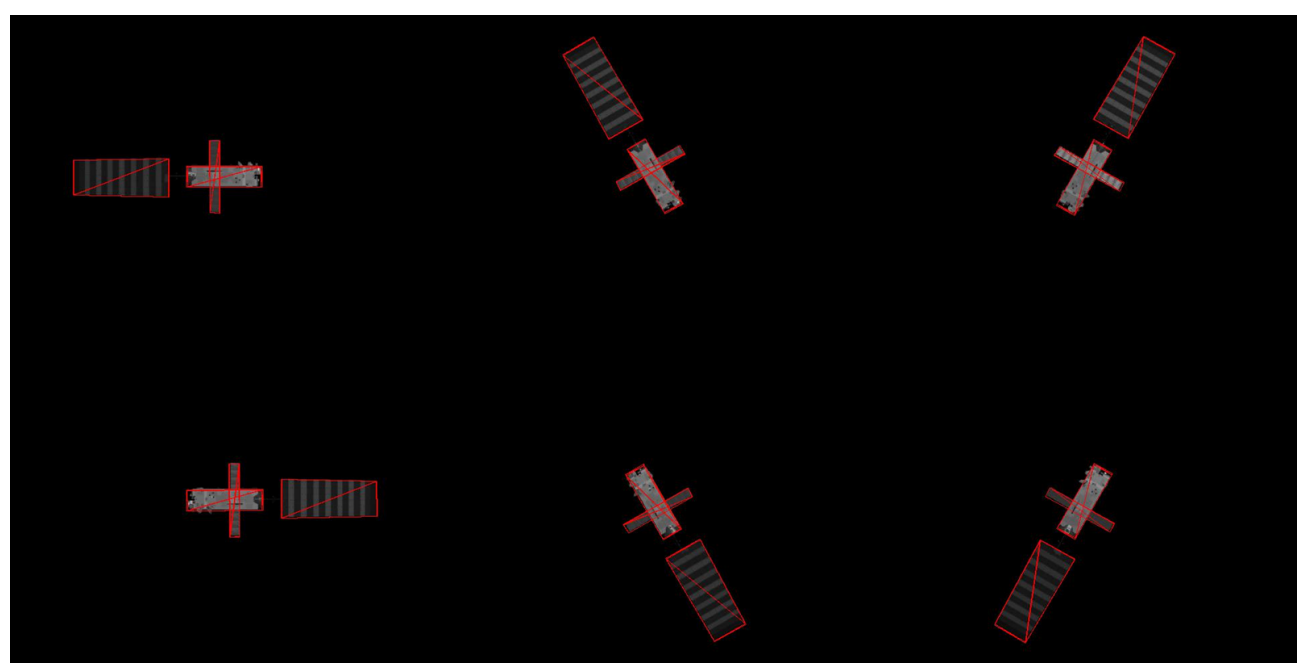

Fig. 8: Pose estimation results for multiple image frames from an Envisat rotation (https://youtu.be/8Km-FOmC8E).

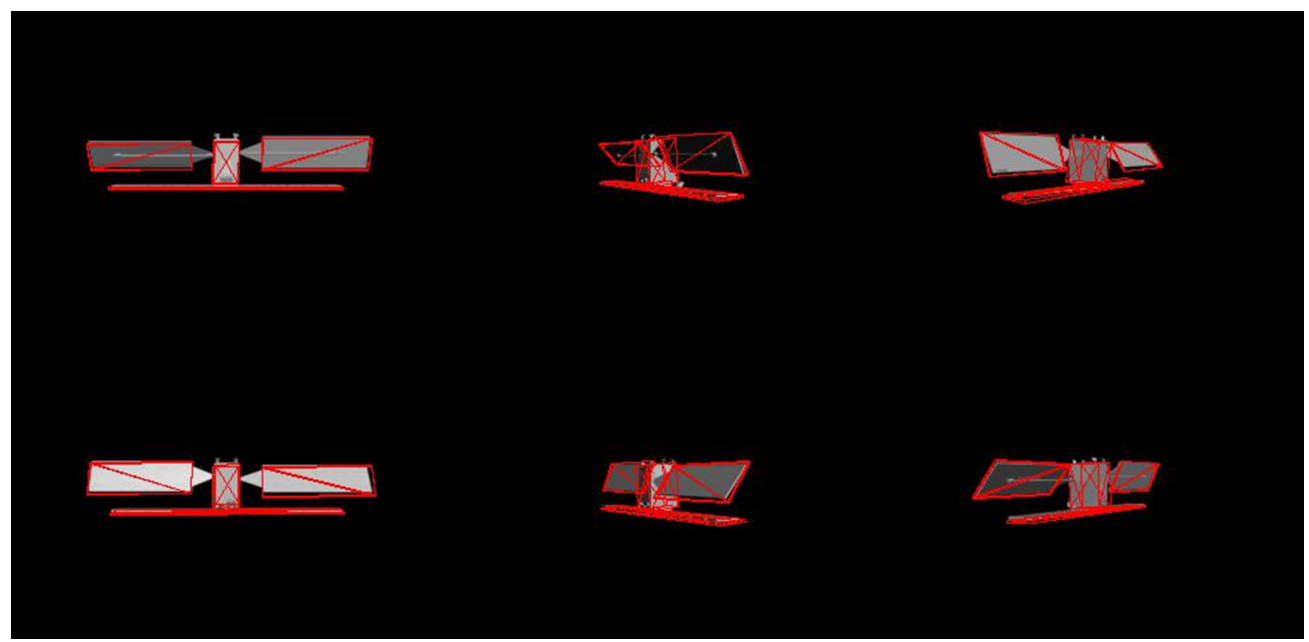

Fig. 9: Pose estimation results for multiple image frames from a Radarsat model rotation (https://youtu.be/IEMpdNHJwic).

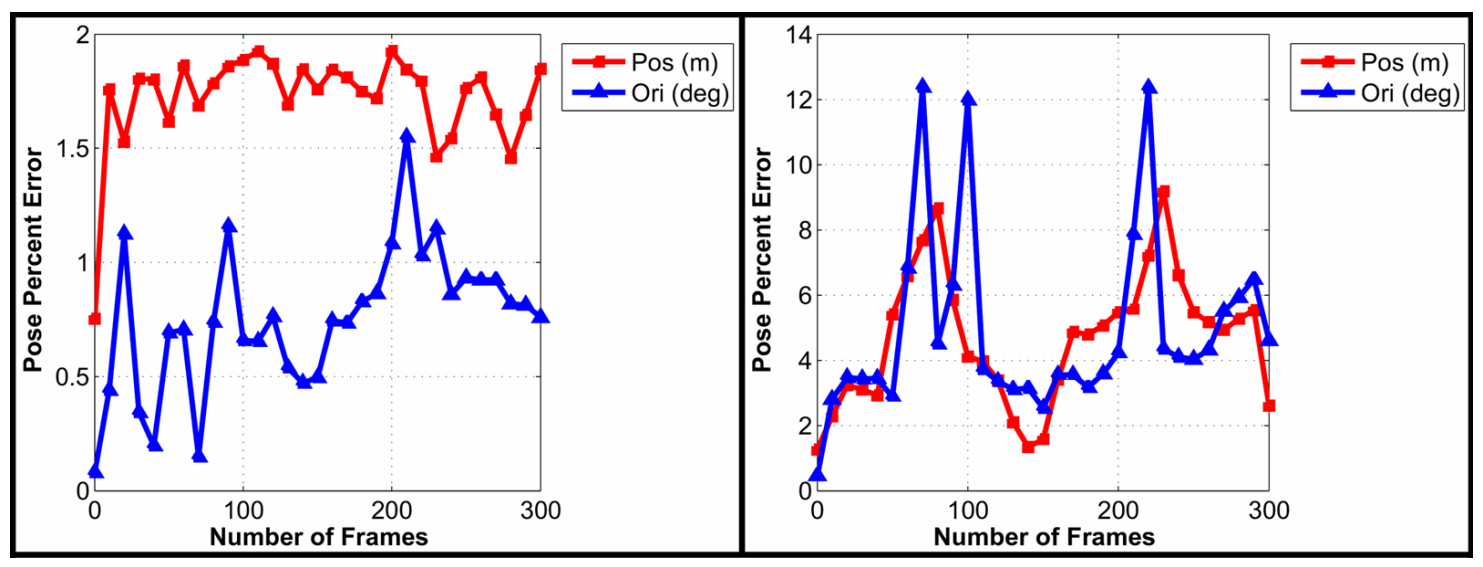

Fig. 10: Pose estimation percentage error. Left: Envisat trial. Right: Radarsat model trial.

\section{Conclusion}

In conclusion, this investigation applies level-set region-based pose estimation by extracting pixel likelihood from the shape prior. A method of refining the foreground posterior map was developed using the Otsu thresholding and image fill 
techniques. The level-set pixel likelihood based pose estimation is a promising tool to overcome complex images in a cluttered scene. Future improvements of this method include improvements to the orientation precision within the foreground map and to classify grayscale images with inseparable pixel intensity distributions.

\section{Acknowledgments}

This research was jointly funded by the NSERC CGSD3-453738-2014, CSA STDP and the OCE VIP-II Award 24053.

\section{References}

[1] V. Lepetit and P. Fua, "Monocular model-based 3d tracking of rigid objects: A survey," Foundations and Trends in Computer Graphics and Vision, vol. 1, no. 1, pp. 1-89, 2005.

[2] B. Rosenhahn, Pose Estimation Revisited, Technical Report 0308, Christian Albrechts-Universität zu Kiel Institut für Informatik und Praktische Mathematik, 2003, http://www.ks.informatik.uni-kiel.de.

[3] B. Rosenhahn, C. Perwass, and G. Sommer, "Foundations about 2d-3d pose estimation," in CVonline: The Evolving Distributed, Non-Proprietary, On-line Compendium of Computer Vision, Edinburgh, 2004.

[4] C. Harris and M. Stephens, "A combined corner and edge detector," Vision Conf., Proc. of the 4th Alvey, vol. 15, pp. 147-151, 1988.

[5] E. Rosten and T. Drummond, "Machine learning for high-speed corner detection," in ECCV, 2006, pp. 430-443.

[6] D. Lowe, "Distinctive image features from scale-invariant keypoints," IJCV, vol. 60, no. 2, pp. 91-110, 2004.

[7] P. Alcantarilla, J. Nuevo, and A. Bartoli, "Fast explicit diffusion for accelerated features in nonlinear scale spaces," in BMVC, 2013.

[8] C. Choi and H. Christensen, "Rgb-d object pose estimation in unstructured environments," Robotics and Autonomous System, vol. 75, pp. 595-613, 2016.

[9] M. Kass, A. Witkin, and D. Terzopoulos, “Snakes: Active contour models," IJCV, vol. 1, no. 4, pp. 321-441, 1988.

[10] D. Mumford and J. Shah, "Optimal approximations by piecewise smooth functions and associated variational problems," Commun. Pure Appl. Math, vol. 42, pp. 577-685, 1989.

[11] V. Caselles, R. Kimmel, and G. Sapiro, "Geodesic active contours," IJCV, vol. 22, no. 1, pp. 61-79, 1997.

[12] T. Chan and L. Vese, "Active contours without edges," in Image Processing, IEEE Transactions on, 2001, pp. 266-277.

[13] S. Osher and J. Sethian, "Fonts propagating with curvature-dependent speed: algorithms based on hamilton-jacobi formulations," JCP, vol. 79, no. 1 , pp. $12-49,1988$.

[14] C. Kervrann and F. Heitz, "Statistical deformable model-based segmentation of image motion," Image Processing, IEEE Trans. on, vol. 8, no. 4, pp. 583-588, 1999.

[15] S. Zhu, L. T.S., and A. Yuille, "Region competition: Unifying snakes, region growing, energy /bayes/mdl for multi-band image segmentation," Pattern Analysis and Machine Intelligence (PAMI), IEEE Trans. on, vol. 18, no. 9, pp. 884-900, 1996.

[16] M. Leventon, W. Grimson, and O. Faugeras, "Statistical shape influence in geodesic active contours," in CVPR, vol. 1, Hilton Head Island, SC, 2000, pp. 316-323.

[17] D. Cremers, "Statistical shape knowledge in variational image segmentation," Ph.D. dissertation, Dept. of Mathematics and Computer Science, University of Mannheim, Mannheim, Germany, 2002.

[18] D. Cremers, T. Kohlberger, and C. Schnörr, "Nonlinear shape statistics in mumford-shah based segmentation," in ECCV, 2002, pp. 516-518.

[19] D. Cremers and C. Schnörr, "Motion competition: variational integration of motion segmentation and shape regularization," in Patter Recognition, vol. 2449, 2002, pp. 472-480.

[20] C. Bibby and R. I., "Robust real-time visual tracking using pixel-wise posteriors," in Computer Vision (ECCV), 9th European Conf. on, Berlin, Heidelberg, 2008, pp. 831-844.

[21] T. Brox and J. Weickert, "Level set based image segmentation with multiple regions," Lecture Notes in Computer Science, pp. 415-423, Aug 2004.

[22] B. Rosenhahn, B. T., C. D., and H. Seidel, "A comparison of shape matching methods for contour based pose estimation," Combinatorial Image Analysis, pp. 263-276, 2006.

[23] T. Brox, B. Rosenhahn, J. Gall, and D. Cremers, "Combined region and motion-based 3d tracking of rigid and articulated objects," PAMI, vol. 32, no. 3, pp. 402-415, 2010.

[24] C. Schmaltz and B. Rosenhahn, "Region-based pose tracking with occulusions using 3d models," Machine Vision and Applications, vol. 23, no. 3, pp. 557-577, 2012.

[25] V. Prisacariu and I. Reid, "Pwp3d: Real-time segmentation and tracking of 3d objects," Computer Vision (IJCV), Intl. Journal of, vol. 98, no. 3, pp. 335-354, 2012.

[26] J. Hexner and R. Hagege, "2d-3d pose estimation of heterogeneous objects using a region based approach," Computer Vision (IJCV), Intl. Journal of, vol. 118, no. 1, pp. 95-112, 2016.

[27] H. Tjaden, U. Schwanecke, and E. Schömer, "Real-time monocular pose estimation of 3d objects using temporally consistent local color histograms," in Computer Vision (ICCV). IEEE 11th Intl. Conf. on. IEEE, 2017, pp. 124-132.

[28] S. Dambreville, R. Sandhu, A. Yezzi, and A. Tannenbaum, "A geometric approach to joint 2d region-based segmentation and 3d pose estimation using a 3d shape prior," SIAM, vol. 3, no. 1, pp. 110-132, 2010.

[29] V. Prisacariu and I. Reid, "Pwp3d: Real-time segmentation and tracking of 3d objects," in BMVC, Sept 2009.

[30] H. Tjaden, U. Schwanecke, and E. Schömer, "Real-time monocular segmentation and pose tracking of multiple objects," in Computer Vision (ECCV), 15th European Conf. on. IEEE, 2016, pp. 423-438.

[31] A. Tsai, Y. A., W. Wells, C. Tempany, T. D., A. Fan, E. Grimson, and A. Willsky, "Model-based curve evolution technique for image segmentation," in CVPR, Kauai, Hawaii, 2001, pp. 463-468.

[32] D. Cremers, M. Rousson, and R. Deriche, "A review of statistical approaches to level set segmentation: integrating color, texture, motion and shape," IJCV, vol. 72, no. 2, pp. 195-215, 2007. 